\title{
New constraints on Lyman- $\alpha$ opacity using 92 quasar lines of sight
}

\author{
Sarah E. I. Bosman ${ }^{1,2}$, Xiaohui Fan ${ }^{3,4}$, Linhua Jiang ${ }^{4}$, Sophie Reed ${ }^{1}$, \\ Yoshiki Matsuoka ${ }^{5}$, George Becker ${ }^{6}$ and Alberto Rorai ${ }^{1,2}$ \\ ${ }^{1}$ Institute of Astronomy, University of Cambridge, Madingley Road, Cambridge CB3 0HA, \\ U.K. \\ ${ }^{2}$ Kavli Institute for Cosmology, University of Cambridge, Madingley Road, Cambridge CB3 \\ OHA, U.K. \\ ${ }^{3}$ Steward Observatory, University of Arizona, Tucson, AZ 85721-0065, USA \\ ${ }^{4}$ Kavli Institute for Astronomy and Astrophysics, Peking University, Beijing 100871, China \\ ${ }^{5}$ National Astronomical Observatory of Japan, Mitaka, Tokyo 181-8588, Japan \\ ${ }^{6}$ Department of Physics \& Astronomy, University of California, Riverside, 900 University \\ Avenue, Riverside, CA, 92521, USA
}

\begin{abstract}
The large scatter in Lyman- $\alpha$ opacity at $z>5.3$ has been an ongoing mystery, prompting a flurry of numerical models. A uniform ultra-violet background has been ruled out at those redshifts, but it is unclear whether any proposed models produce sufficient inhomogeneities. In this paper we provide an update on the measurement which first highlighted the issue: Lyman- $\alpha$ effective optical depth along high- $z$ quasar lines of sight. We nearly triple on the previous sample size in such a study thanks to the cooperation of the DES-VHS, SHELLQs, and SDSS collaborations as well as new reductions and spectra. We find that a uniform UVB model is ruled out at $5.1<z<5.3$, as well as higher redshifts, which is perplexing. We provide the first such measurements at $z \sim 6$. None of the numerical models we confronted to this data could reproduce the observed scatter.
\end{abstract}

Keywords. quasars: absorption lines, cosmology: observations, large-scale structure of universe

\section{Introduction}

Distant accreting black holes act as flash-lights illuminating an evolving Universe. In the past decade, the lines of sight to the most distant quasars have become foremost tools in observational cosmology and the analysis of galaxy formation thanks to their powerful black body radiation continuum.

High redshift quasar lines of sight gave the first hints on the timing of reionisation, narrowing down the time at which the Universe became transparent to UV light to $z>6.0$ (e.g. Fan et al. 2001, Fan et al. 2006). The same lines of sight offer the most precise measurements of cosmic metal enrichment, which remains a puzzle in the era of galaxy formation (e.g. Simcoe et al. 2011, Keating et al. 2016, Bosman et al. 2017). With the discovery of the first bright $z>7.0$ quasar in 2011, controversial hints of a highly neutral IGM were noticed (Mortlock et al. 2011, Bosman \& Becker 2015, Greig et al. 2017). Perhaps the most surprising observation to have come from quasar lines of sight was made in 2015: the discovery that the ionisation of the Universe is very inhomogeneous even at $z \sim 5.5$ when reionisation is expected to have already all but completed.

Until the deep X-Shooter observations of the $z=6.0$ quasar J0148+0600 by Becker et al. 2015, the observed opacity of Lyman- $\alpha$ absorption at high redshift was (perhaps 


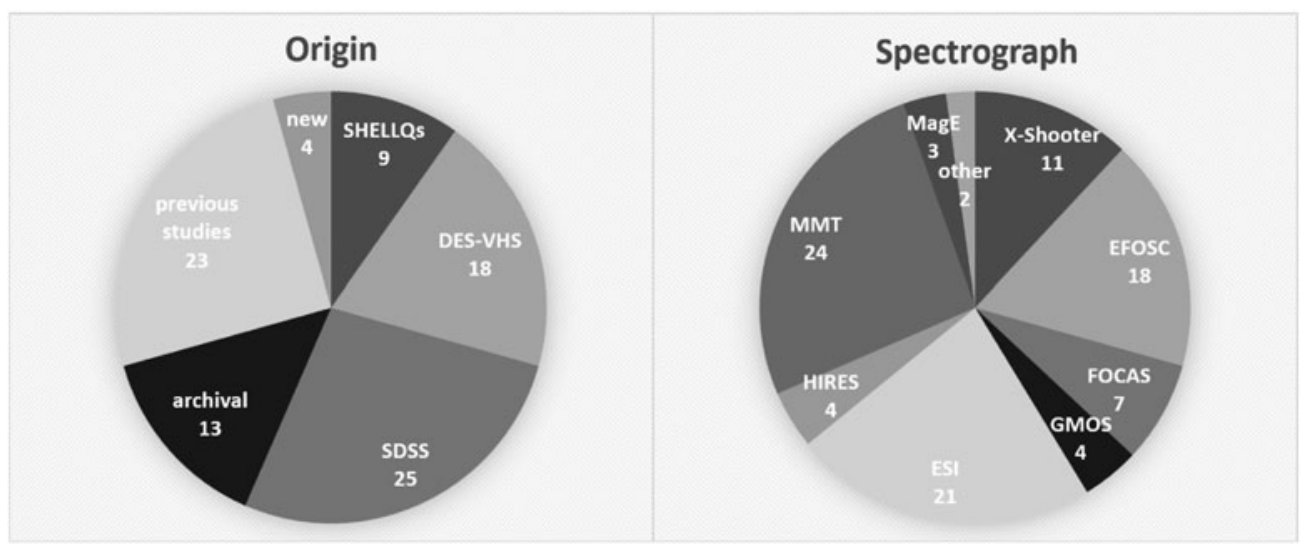

Figure 1. Provenance (left) and spectrograph of origin (right) of the 92 quasar lines of sight at $z>5.7$ in our sample.

marginally†) consistent with a smooth ultra-violet background (UVB), with increasing opacity at earlier times pointing towards a smooth weakening of the background with redshift. It was therefore highly unexpected to find that stretches of the IGM as long as $\sim 110 \mathrm{cMpc} h^{-1}$ showed total Lyman- $\alpha$ absorption down to $z=5.5$, while many others already display ample transmission. It was quickly established that models featuring a uniform UVB could not reproduce this; in other words, UV photon percolation has not completed yet at $z=5.5$.

Three families of models emerged to ease the problem, with moderate results. Roughly speaking, the physical motivations behind those models are as follows:

(a) Chardin et al. (2015) tested the hypothesis that the opacity inhomogeneity could be reproduced if rare bright sources were significant contributors to reionisation.

(b) D'Aloisio et al. (2015) tested the inclusion of radiative transfer into numerical simulations, as the resulting temperature fluctuations give rise to differential timing of reionisation.

(c) Davies \& Furlanetto (2016) proposed that the inhomogeneities arise naturally from fluctuations in the UVB due to a short and varying mean free path of ionising photons.

It still remains unclear which of these models, if any, give a satisfactory fit to Lyman$\alpha$ transmission data. While other implications of these models and new avenues for comparison are being investigated (see e.g. Chardin et al. 2017, Davies et al. 2017), in our work we aim to provide an update on the observations which first prompted their creation: the distribution of Lyman- $\alpha$ opacity along quasar lines of sight.

\section{Overview}

More than 250 bright quasars have now been found at $z>5.7$, and more than 120 at $z>6.0 \ddagger$. However, the largest compilations of these objects in a single study have been limited in size to less than 40 (Becker et al. 2015, Eilers et al. 2017). Bringing together spectra from many different authors as well as discovery spectra from multiple collaborations, we have increased this number to 92 quasar lines of sight. For the first

$\dagger$ A handful of authors worried about the discrepancies between lines of sight as early as 2006 (see Lidz et al. 2006, Bolton \& Haehnelt 2007).

$\ddagger$ For an up-to-date list, refer to http://www.ast.cam.ac.uk/ seib2/list_of_all_quasars.htm 


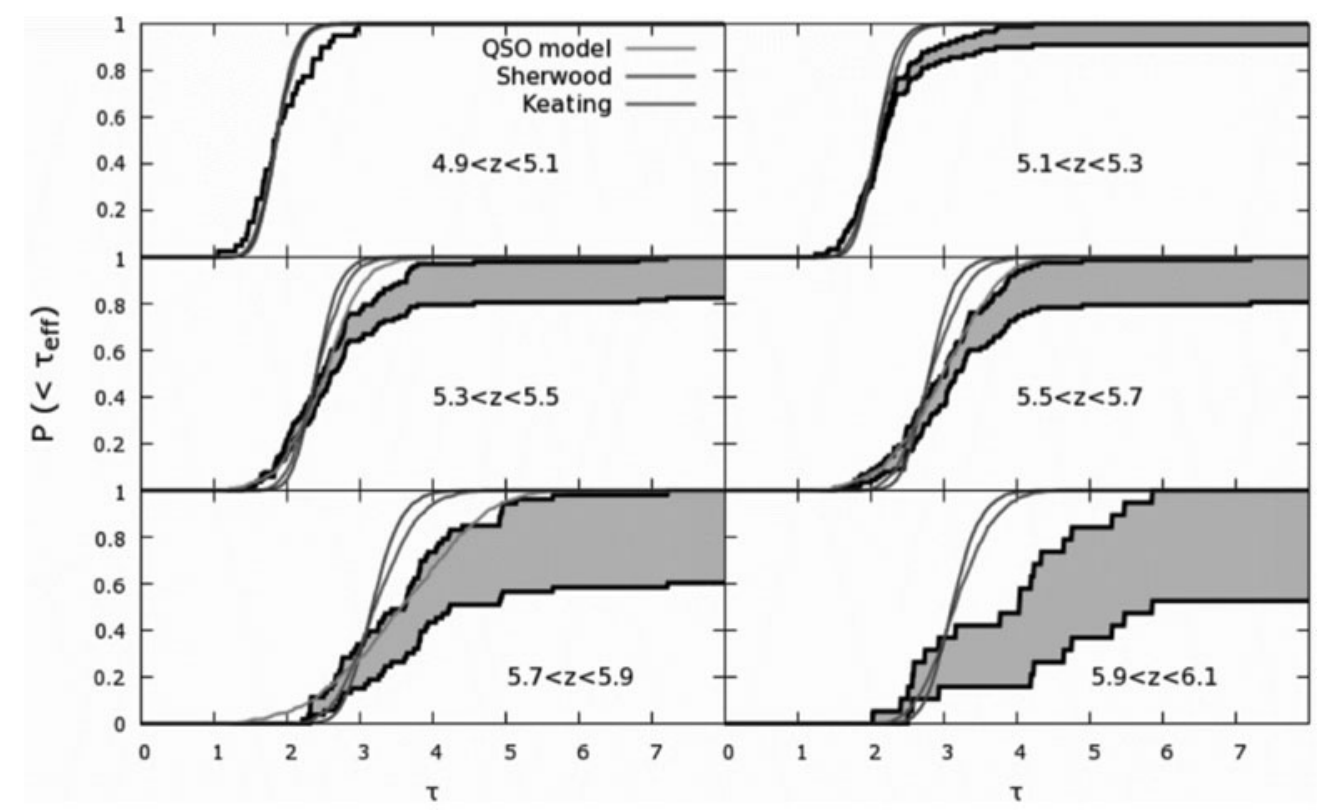

Figure 2. Lyman- $\alpha$ opacity along quasar lines of sight at $4.9<z<6.1$. Three outputs from numerical simulations are shown, after rescaling their emissivity to match the average flux.

time, this allows up to quantise the scale of cosmic variance between objects as well as extend the measurements to higher redshifts.

This was made possible thanks to the cooperation of the SDSS (25 spectra), DES-VHS (18 spectra) and SHELLQs (9 spectra) collaborations, as well as the authors of previous such studies (23 spectra; Becker et al. 2015, McGreer et al. 2015). Finally, 13 spectra were reduced from pre-existing archival data, and 4 are new to this work. Overall, the data originates from ten different optical spectrographs with a range of wavelength coverages, resolutions, and exposure times (see Figure 1).

The spectra were cleaned in preparation for the measurement of opacity in the standard way: the continuum is modelled by a power-law fit with multiple rounds of sigma clipping, bad pixels are removed, and DLAs were detected via their associated metal absorption lines and excluded from the sample. Careful attention was paid to the effect of cutting out the quasars' proximity zones in a consistent way. After running the whole analysis with different values of proximity zone cut-offs, and analysing spectral stacks, a fixed final threshold of $\lambda=1178 \AA$ was chosen (a value adopted by multiple previous studies).

Effective Lyman- $\alpha$ optical depth was measured in bins of $50 \mathrm{cMpc} h^{-1}$ following $\tau_{\text {eff }}=$ $-\ln \left(\langle F\rangle_{\text {bin }}\right)$. The values of optical depths are then plotted as cumulative distribution functions (CDFs). The measurements were repeated with bin sizes of 10, 30 and 70 $\mathrm{cMpc} h^{-1}$. We find a strong shift towards more opacity scatter for smaller bins, and no statistically significant difference between the 50 and $70 \mathrm{cMpc} h^{-1}$ cases. We also investigate the effect of restricting the analysis to lines of sight of high spectral quality, finding the results are consistent.

We introduce a new way of quantifying the 'allowed regions' of the opacity distribution, by using bounds determined by the 'most optimistic' and 'most pessimistic' interpretations of non-detections of transmission. The 'optimistic' case, following previous work, assigns a value of twice the error to non-detections; i.e. the real flux is considered to lie just below the detection threshold. The 'pessimistic' case considers non-detections to 
indicate maximal opacity, i.e. $F=0, \tau \rightarrow \infty$. As long as the sample size is sufficient to resolve these bounds, any predictions from models must therefore necessarily lie in between these bounds.

\section{Implications}

Our results are statistically consistent with previous studies as confirmed by a bootstrap analysis. The tail of the distribution towards high opacities is confirmed and resolved in much better detail at $5.3<z<5.9$, and we also find evidence of an opaque tail at $5.1<z<5.3$ which had not been previously reported. This result is unexpected since percolation of UV photons is predicted to have happened by this time by all models. In addition, we produce the first Lyman- $\alpha$ opacity CDF at $5.9<z<6.1$, finding a very high scatter in opacities with higher average opacity than lower redshifts.

We compare the results with simulated lines of sight from three simulations - the Sherwood simulation which uses a smooth UVB (Bolton et al. 2017), the simulations of Chardin et al. (2015) implementing rare bright sources as the cause of reionisation, and the simulations from Keating et al. (2017) with full radiative transfer effects which test the viability of scenario (b) above. We find that none of these models accurately reproduce the shape of the Lyman- $\alpha$ opacity distribution, even after the mean fluxes in the simulation outputs are tuned to observations. Out of the three models we test, the rare sources model comes the closest to the observed distribution at $z>5.5$. However, photon percolation happens rapidly in that model, and not enough opaque lines of sight survive at $5.1<z<5.5$.

In light of these results, the extreme scatter of Lyman- $\alpha$ opacity at the tail end of reionisation remains a perplexing puzzle. Recent investigations of the reionisation epoch have highlighted the importance of self-shielding mechanisms, as well as early X-ray heating of the IGM. Implementing such effects in numerical simulations is tricky, but might prove necessary to begin to resolve this conflict.

\section{References}

Becker, G. D., Bolton, J. S., Madau, P., et al. 2015, MNRAS, 447, 3402

Bolton \& Haehnelt 2007, MNRAS, 382, 325

Bolton, J. S., Puchwein, E., Sijacki, D., et al. 2017, MNRAS, 464, 897

Bosman, S. E. I. \& Becker, G. D. 2015, MNRAS, 452, 1105

Bosman, S. E. I., Becker, G. D., Haehnelt, M. G., et al. 2017, MNRAS, 470, 1919

Chardin, J., Haehnelt, M. G., Aubert, D., \& Puchwein, E. 2015, MNRAS, 453, 2943

Chardin, J., Haehnelt, M. G., Bosman, S. E. I., \& Puchwein, E. 2017, arXiv:1707.03841

D'Aloisio, A., McQuinn, M., \& Trac, H. 2015, ApJL, 813, L38

Davies, F. B. \& Furlanetto, S. R. 2016, MNRAS, 460, 1328

Davies, F. B., Becker, G. D., \& Furlanetto, S. R. 2017, arXiv:1708.08927

Eilers, A.-C., Davies, F. B., Hennawi, J. F., et al. 2017, APJ, 840, 24

Fan, X., Narayanan, V. K., Lupton, R. H., et al. 2001, AJ, 122, 2833

Fan, X., Strauss, M. A., Becker, R. H., et al. 2006, AJ, 132, 117

Greig, B., Mesinger, A., Haiman, Z., \& Simcoe, R. A. 2017, MNRAS, 466, 4239

Keating, L. C., Puchwein, E., Haehnelt, M. G., Bird, S., \& Bolton, J. S. 2016, MNRAS, 461, 606

Keating, L. C., Puchwein, E., \& Haehnelt, M. G. 2017, arXiv:1709.05351

Lidz, A., Oh, S. P., \& Furlanetto, S. R. 2006, ApJL, 639, L47

McGreer, I. D., Mesinger, A., \& D'Odorico, V. 2015, MNRAS, 447, 499

Mortlock, D. J., Warren, S. J., Venemans, B. P., et al. 2011, Nature, 474, 616

Simcoe, R. A., Cooksey, K. L., Matejek, M., et al. 2011, APJ, 743, 21 